\title{
El campo petrolero Cantarell Y LA ECONOMÍA MEXICANA
}

\author{
Daniel Romo ${ }^{1}$ \\ Fecha de recepción: 5 de enero de 2015. Fecha de aceptación: 20 de abril de 2015.
}

\begin{abstract}
RESUMEN
Los grandes campos de petróleo han jugado un papel fundamental como aporte de hidrocarburos en el mundo, pero la mayoría de ellos está en proceso de declinación. El macro yacimiento Cantarell ha sido el origen de importantes volúmenes de hidrocarburos y grandes beneficios para el país, pero a partir de 2004 inició su proceso de declinación que ha afectado la plataforma de producción de petróleo. Esta investigación estudia el conjunto de elementos que han incidido sobre el desempeño de ese macro yacimiento, los cuales son fundamentales para explicar su situación actual. Se concluye que la administración de Cantarell no fue la óptima durante los tres periodos característicos identificados, toda vez que se sujetó a determinaciones no técnicas, que incidieron en su declinación y en la serie de retos que está enfrentando, mismos que a su vez, podrían atenuarse con el empleo de tecnología.
\end{abstract}

Palabras clave: petróleo, Cantarell, hidrocarburos, Pemex, inversión.

Clasificación JEL: L32, N76, O13, Q43, Q48.

\section{The Cantarell Oil Field AND THE MEXICAN ECONOMY}

\begin{abstract}
Major oil fields have played an essential role in the global hydrocarbon supply, but the majority are now in decline. The Cantarell macro-reserve has produced significant volumes of hydrocarbons and brought countless benefits to the country, but starting in 2004, the exhaustion of the Cantarell supply began to affect the oil production platform. This research studies all of the factors that have had an impact on the performance of this macro-reserve, which are fundamental to explain the current state of affairs. The conclusion is that the management of the Cantarell oil field was less than optimal during the three time periods identified in the paper, as decisions were made on a nontechnical basis, which contributed to the decline and the series of challenges facing the oil complex today. These difficulties could, in turn, be mitigated through the use of technology.
\end{abstract}

Key Words: Oil, Cantarell, hydrocarbons, Pemex, investment.

${ }^{1}$ Instituto Politécnico Nacional, México. Correo electrónico: dromor@ipn.mx 


\section{LE CHAMP PÉTROLIFÈRE CANTARELL ET L'ÉCONOMIE MEXICAINE Résumé}

Les grands champs pétrolifères du Mexique ont joué un rôle fondamental dans la fourniture d'hydrocarbures dans le monde, mais la plupart d'entre eux sont en déclin. Le macro-gisement Cantarell a prodigué d'importants volumes d'hydrocarbures et de grands bénéfices pour le pays, mais depuis 2004 il est entré dans son déclin affectant la plateforme de production de pétrole. Cette recherche se penche sur l'ensemble des éléments qui ont eu une incidence sur les performances de ce macro-gisement, lesquels sont essentiels pour expliquer sa situation actuelle. Il en est tiré la conclusion que la gestion de Cantarell n'a pas été optimum durant les trois périodes caractéristiques identifiées, dans la mesure où elle a été soumise à des facteurs non techniques, qui ont joué un rôle dans son déclin et dans la série de défis auxquels il été confronté, lesquels pourraient eux-mêmes être atténués par l'emploi de technologie.

Mots clés: pétrole, Cantarell, hydrocarbures, Pemex, investissement.

\section{O CAMPO PETROLEIRO CANTARELL E A ECONOMIA MEXICANA}

\section{Resumo}

Os grandes campos de petróleo tiveram um papel fundamental como contribuição de hidrocarbonetos no mundo, mas a maioria deles está em processo de diminuição. O macro- aurífero Cantarell foi a origem de importante volume de hidrocarbonetos e de grandes lucros para o país, mas a partir de 2004 iniciou seu processo de declínio que afetou a plataforma de produção de petróleo. Esta pesquisa estuda o conjunto de elementos que incidiram no desempenho atual desse macro-aurífero, os quais são fundamentais para explicar a sua situação atual. A conclusão é que a administração de Cantarell não foi ótima durante os três períodos característicos identificados, já que se sujeitou a determinaçóes não-técnicas, que incidiram no seu declínio e na serie de desafios que está enfrentando, estes que poderiam ser atenuados com o emprego de tecnologia.

Palavras-chave: Cantarell, hidrocarbonetos, Pemex, investimento.

坎塔雷尔油田和, 墨西哥经济

大型油田是碳氢化合物的重要来源, 但是目前大部分油田处于衰竭阶段。 坎塔雷尔油田曾产出大量碳氢化合物为墨西哥带来了巨大的利益。但是自 2004年起该油田开始枯竭, 对石油生产平台产生了影响。本文对影响该 油田运行的因素进行研究。研究结论为坎塔雷尔油田在三个阶段中的管理 并不完善, 很多决定没有考虑到技术因素, 这导致了它的衰竭以及现在面 临的一系列挑战, 而这些挑战可以通过技术进行缓解。

关键词: 石油, 坎塔雷尔, 碳氢化合物, 墨西哥国家石油公司, 投资 


\section{INTRODUCCIÓN}

Los grandes campos de petróleo han sido base de aportación en el mundo. Empero, muchos de ellos han entrado en una etapa de declinación, misma que ha llevado a buscar alternativas para prolongar su vida útil o encontrar alternativas para satisfacer la demanda de petróleo, como ha sido la explotación de los crudos no convencionales.

Cantarell ha sido considerado uno de los 100 más importantes campos petroleros en el mundo que llegó a contribuir con el $45 \%$ de la producción mundial (Robelius, 2007: 77), y forma parte del grupo de campos en proceso de declinación (Sorrel et al., 2012: 710). También fue el punto de origen de la extracción de los mayores volúmenes de petróleo en México desde fines de los años setenta; sin embargo, a partir de los últimos meses de 2004 inició un proceso de agotamiento que lo llevó a producir a mediados de ese año sólo el $15.4 \%$ del total máximo de producción histórica alcanzado en diciembre de 2003. Ante tal comportamiento, surge la pregunta sobre la racionalidad en la velocidad de declinación. Algunos trabajos se han realizado para explicar el desempeño del macro yacimiento desde el punto de vista técnico, incluidos los elaborados por Pemex, y otros con un enfoque más institucional (Lajous, 2009), pero no han explorado un análisis de tipo estructural ligado al entorno económico del país y su influencia en las determinaciones sobre su administración.

Este trabajo tiene el objetivo de analizar el desempeño de Cantarell a través del tiempo y su rol, como parte fundamental de la plataforma de producción petrolera en el desenvolvimiento económico del país. El documento está organizado en cuatro secciones. En la primera, se hace un recuento de los principales factores que incidieron en su desempeño a través del tiempo. En la segunda, se hace un recuento de su situación en el pasado reciente, con el objetivo identificar los principales elementos que explican su situación actual. En la tercera sección, se analizan sus principales retos y perspectivas. Finalmente, se concluye que Cantarell ha sido el origen de importantes recursos financieros que han apoyado el crecimiento económico, pero que su administración fue poco eficiente debido a una serie de decisiones que se privilegiaron sobre una visión de largo plazo, lo cual fue relevante en su rápida declinación y en los retos que ahora enfrenta. 


\section{CANTARELL Y LOS FACTORES QUE INFLUYERON EN SU DESEMPEÑO}

Cantarell es la denominación que en Petróleos Mexicanos (Pemex) ha dado a un conjunto de campos ubicados en el área marina del estado mexicano de Campeche, que abarcan una superficie de alrededor de 21 mil kilómetros cuadrados. Ha sido de los campos productores que más volúmenes de hidrocarburos y de renta petrolera han aportado al país.

Los trabajos de reconocimiento comenzaron en el mar desde 1966, pero fue hasta 1972 cuando se concluyó sobre la prolongación de los yacimientos terrestres de los estados de Chiapas y Tabasco, a pesar de que los costos exploratorios y de perforación sextuplicaban a los continentales. En una primera etapa se delinearon las estructuras del campo Chac. ${ }^{2}$ La explotación de estos campos inició el 23 de junio de 1979 en el pozo descubridor de aceite denominado Cantarell 2095, el cual tenía una columna impregnada de 974 metros. En ese año, se obtuvo en el campo Akal una producción promedio de 42 mil barriles diarios (mbd) por pozo e inició la producción en el campo Nohoch con un volumen de $4.3 \mathrm{mbd}$ y se descubrió el campo Kutz. Estos eventos ocurrieron en un entorno de elevados precios del crudo por los problemas políticos en Irán y las disputas entre los miembros de la Organización de Países Exportadores de Petróleo (ореP), entre otros factores, situación que alentó la actividad petrolera en el país.

La producción inicial de petróleo en Cantarell promedió 88 mbd en 1979, pero para 1980 subió hasta $611 \mathrm{mbd}$, gracias a los avances en la caracterización estructural de la plataforma de Campeche. En 1981 se alcanzó un máximo de producción de petróleo de 1,156 mbd mediante la explotación de tan sólo 40 pozos, básicamente empleando la inyección de agua en el yacimiento. Las condiciones de los elevados precios del petróleo crudo, debido básicamente a las consecuencias de los cortes en la oferta del crudo por el conflicto Iraní-Iraquí, fueron los principales móviles para que Pemex elevará el volumen de crudo producido y se dinamizaron muchas inversiones en toda la cadena de valor de la industria nacional.

En 1982, el país enfrentó problemas de liquidez y el gobierno un abultado déficit en las finanzas públicas, empero la plataforma de producción aumentó

En septiembre de 1974 la perforación alcanzó a una profundidad de 3400 metros identificándose sedimentos saturados de petróleo, con porosidad y permeabilidad. En 1975 se continuó la perforación hasta 3535 metros, encontrándose impregnaciones de petróleo, por lo cual se perforó un segundo pozo durante 1976 hasta los 4934 metros. 
a 1.6 millones de barriles por día (mmbd) en un mercado petrolero internacional sobreofrecido. En 1983, se le impuso a Pemex la obligación de auxiliar al fisco con pago de impuestos, la recaudación del impuesto sobre el valor agregado y del impuesto especial sobre producción y servicios, de manera tal que sus contribuciones fiscales pasaron de $16 \%$ en 1981 a $43 \%$ del total de ingresos públicos en ese 1983. El entorno de ajustes al gasto público y los esfuerzos por reducir su déficit fiscal, impactaron el nivel de inversión de la petrolera estatal (Romo, 2010: 417-444). No obstante, dentro de su cartera de inversiones, se privilegiaron las operaciones de Cantarell, particularmente la ampliación del número de pozos productores.

En mayo de 1984, inició la explotación del campo Ixtoc con un promedio de producción de 5.2 mbd. Además en 1987, se llevó a cabo la colocación de sistemas artificiales de explotación (el bombeo neumático), que dieron como resultado mantener la plataforma de producción en un promedio de $950 \mathrm{mbd}$. En esos años, la producción de petróleo contribuyó a apoyar el desempeño de la debilidad financiera registrada en el país y en las finanzas públicas en particular.

A mediados de los años ochenta, el gobierno inició un proceso de apertura al exterior, seguido de la privatización de empresas públicas y la desregulación de la economía en los años siguientes. También se preservaron los ajustes en el gasto público a fin de reducir su déficit financiero y los escasos recursos de inversión a Pemex, situación que propició un proceso de ajuste en el portafolio de sus actividades. Se bajó el ritmo de las operaciones de exploración y se dio limitada atención a las nuevas inversiones en refinación y petroquímica, dándole preponderancia a la explotación de petróleo, en donde destacaron las operaciones en Cantarell. El cambio en el modelo de negocio en Pemex tuvo como marco la sobreoferta de crudo enfrentada en el mercado petrolero internacional (Romo, 2011: 140).

En 1990, México llevó a cabo una favorable renegociación de su deuda externa, que redujo las presiones financieras del gobierno y de los pasivos de la petrolera estatal, también incluidos en esa renegociación. No obstante, las inversiones canalizadas a Pemex sólo se recuperaron ligeramente, siendo Cantarell uno de los principales destinos de tales recursos. Entre 1991 y 1994, las inversiones hacia el macro yacimiento concentraron alrededor de una quinta parte del total de erogaciones en exploración y producción de Pemex, siendo su principal objetivo la incorporación de reservas, mantener la plataforma de producción, incrementar la capacidad de deshidratación de gas, de su transporte y a terminar la plataforma de perforación Nohoch C. En agosto de 
1991, se logró iniciar la producción del campo Chac y en febrero de 1993 la explotación del campo Balam, que mostró cambios notables en su producción en esos primeros años. ${ }^{3}$

Con el estallamiento de la crisis de finales de 1994, la situación financiera del gobierno se volvió a afectar y nuevos ajustes en el gasto público se instrumentaron, pero al contrario de lo que sucedió en los años previos, se canalizó adicional inversión en explotación, y en especial a Cantarell. El objetivo fue respaldar el compromiso pactado con los acreedores internacionales, en donde el petróleo se otorgó como garantía de los préstamos obtenidos y ampliar el flujo de petrodivisas. En 1995, Pemex mantuvo una plataforma de producción por arriba del $1.0 \mathrm{mmbd}$ alcanzada desde 1990, sin embargo, ante el aumento de la inversión, llegó a promediar 1.3 mmbd entre 1997 y 1999.

En 1996, Pemex llevó a cabo el Proyecto de Optimización de Cantarell (POC), con el fin de mantener la capacidad productiva de los pozos y de reducir sus costos de mantenimiento, mismo que se sustentó con el mayor involucramiento de las empresas privadas. ${ }^{4}$ Hacia el año siguiente, se instrumentaron los llamados Proyecto de Inversión de Infraestructura Productiva con Registro Diferido en el Gasto Público (pidiregas), cuyo objeto fue el canalizar recursos adicionales a los proyectos estratégicos de la petrolera estatal. Uno de ellos fue el Proyecto Cantarell orientado a mejorar su infraestructura y mantener su presión para elevar su producción ${ }^{5}$ mediante la inyección de nitrógeno. La justificación de esta última determinación se basó en que la energía del yacimiento estaba dominada por el casquete de gas secundario y se pensó que para aumentar la producción se necesitaba más energía a través de dicha inyección. Tal determinación fue polémica, pues si bien se eligió por los beneficios económicos, ante el bajo precio del nitrógeno y la posibilidad evitar las importaciones de gas natural (Lajous, 2009), fue criticada desde el punto de vista técnico que propuso la reinyección de gas natural (Garaicoechea, 2007: 4-6).

Después de iniciar con una producción de $3.7 \mathrm{mbd}$, el campo Balam alcanzó una plataforma de 55 mbd en diciembre de 1994, pero el año siguiente sólo promedio $20.3 \mathrm{mbd}$, manteniendo esa tendencia en 1996, para luego llegar a tocar un mínimo de sólo 6.9 mbd en mayo de 1997.

4 En 1997 se contrataron también los servicios de Bechtel para participar en la administración del proyecto y se desarrollaron nuevas formas de contratación y adquisición de materiales y equipos acordes con las "prácticas internacionales".

5 Entre 1997 y 1998, las estrategias contemplaron incrementar la producción de Cantarell a 1.4 millones de barriles por día; reducir el envío de gas a la atmósfera; iniciar la inyección de gas al yacimiento, entre otras. 
En un entorno de precios bajos del petróleo crudo en los mercados internacionales durante 1998, y su proceso de recuperación en los dos años siguientes, las inversiones en Cantarell fueron el objetivo principal de las operaciones de Pemex, junto con la de otros proyectos como para impulsar la producción de gas y petróleo. ${ }^{6}$ En ese 1998, fue descubierto el bloque Sihil, por debajo de Akal, que se anunció con un potencial de más de mil millones de barriles de petróleo crudo equivalente (mmmbpce), algo así como 5\% de las reservas originales del campo Akal (Barton, 2009/2010: 28).

En los primeros cuatro años de operación del esquema PIDIREGAs, se canalizaron un promedio anual de 2 mil millones de dólares ( $\mathrm{mmmd}$ ) al denominado Complejo Cantarell. A principios de 2000, se incluyó el mejoramiento de su infraestructura para optimizar el aprovechamiento de gas y ampliar las operaciones de perforación ${ }^{7}$ e inició la inyección de nitrógeno. A partir de 2002, se amplió el portafolio de proyectos de exploración y explotación de petróleo y gas natural, entre los que destacaron el denominado Integral EkBalam, que se contempló como parte del Complejo Cantarell. Por ello, la inversión anual promedio aumentó a 2.4 mmd entre 2002 y 2007 a 3.8 mmd entre 2008 y 2013, representando los mayores volúmenes canalizados en la petrolera estatal (véase gráfica 1), y teniendo como elemento central el crecimiento en el número de pozos. ${ }^{8}$

En diciembre de 2003, Cantarell alcanzó un pico de producción de 2.2 mmbd, soportado por el campo Akal, que contribuyó con el 95.2\% del total explotado del Complejo (véase gráfica 2). En ese momento, México logró un máximo histórico de producción de crudo del que ese macro yacimiento llegó a aportar casi dos terceras partes del total nacional, e incluso, a considerarse como el número uno de producción costa afuera en el mundo y el segundo más grande de los macro yacimientos productores, sólo detrás del campo árabe Ghawar. Ello fue tan relevante que Cantarell llegó a promediar el 2.3\% de la oferta mundial de petróleo entre los años 2000 y 2003, después de haberlo hecho con $1.4 \%$ entre 1980 y 1999 .

A finales de 2004, la declinación de Cantarell estuvo determinada por el agotamiento del campo Akal, pese a las estrategias instrumentadas, la inver-

Otros proyectos importantes en esos años fueron Burgos y Delta del Grijalva, además de la reconfiguración de la Refinería de Cadereyta.

7 Entre 1997 y 2002, se construyeron 26 plataformas marinas de perforación, de producción y de compresión de gas. Pemex, Boletín de prensa 17/05/2002.

8 Se llegó a contar con 218 pozos en operación en diciembre de 2003, lo que representó casi el 50\% más de los pozos con los que se contaba en 1996. 
Daniel Romo

Gráfica 1. Inversiones en Cantarell y su relación con Pemex

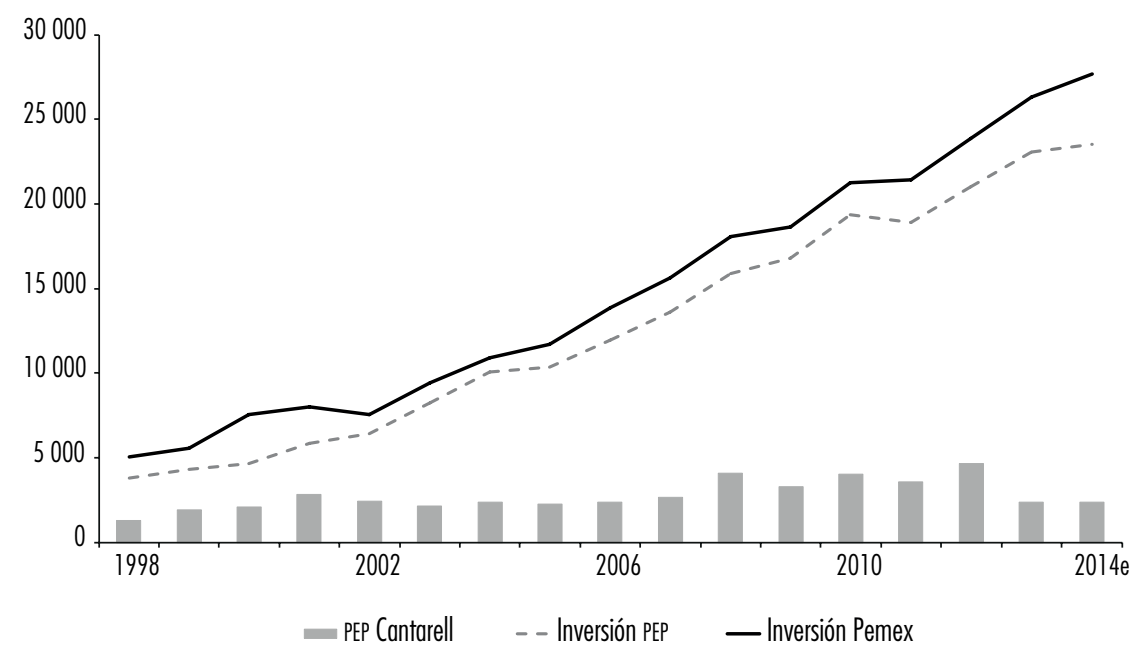

Fuente: elaborado con datos de Pemex.

Grática 2. Producción de petróleo en Cantarell. Miles de barriles por día

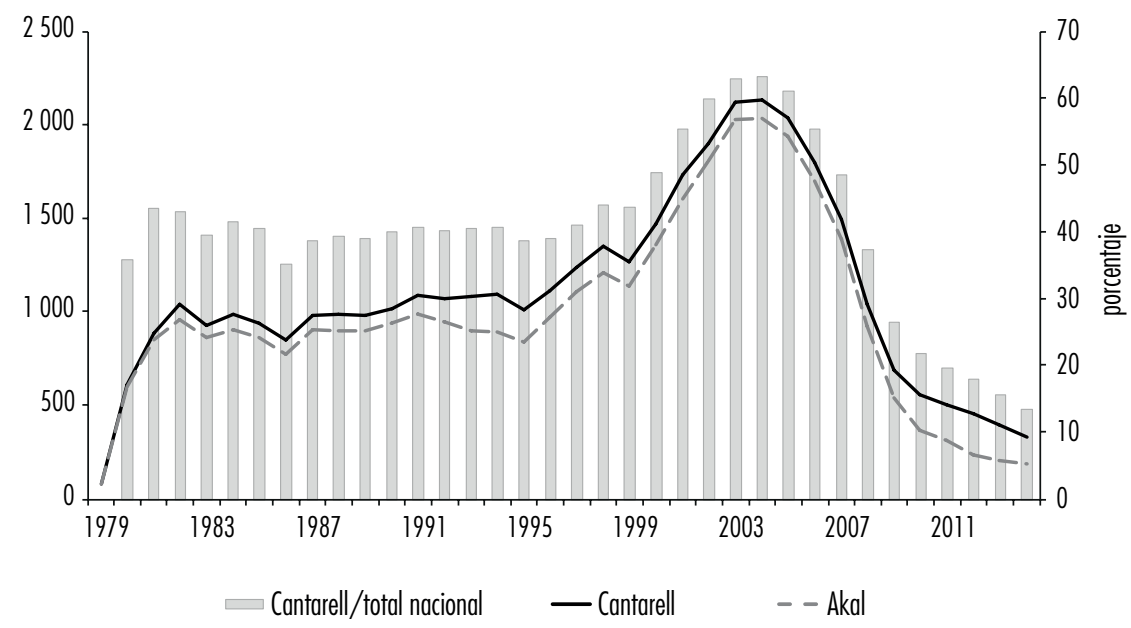

Fuente: elaborado con datos de Pemex. 
sión canalizada y la participación de las empresas privadas. ${ }^{9}$ La declinación en la producción de aceite fue en contrasentido con el incremento en la producción de gas natural entre 2004 y 2009. Un elemento determinante de ese comportamiento, además del reacomodo de fluidos del yacimiento, fue el alza en la producción de nitrógeno, que llegó a representar a mediados de 2014 un poco más de la mitad de la producción total de gas natural. La disminución en la producción de los hidrocarburos producidos en Cantarell fue compensada por el aumento de otros proyectos (Ku-Maloop-Zaap, Litoral Tabasco y ciertos campos de la Región Sur). Durante 2006, Pemex instrumentó adicionales medidas para mantener la presión del macro yacimiento ${ }^{10}$ y evitar la caída en la producción del crudo ${ }^{11}$ sin éxito, pues continúo su declive. En el año 2008, cuando se descubrió el campo Kambesah y los precios internacionales de petróleo alcanzaron máximos históricos, se aprobaron cambios al marco legal aplicado a la petrolera estatal que le permitió agilizar sus operaciones de adquisición de servicios y bienes; ${ }^{12}$ de hacer uso de los Contratos Integrales de Exploración y Producción e implantar la práctica del Gobierno Corporativo. Se creó la Comisión Nacional de Hidrocarburos ( $\mathrm{CNH}$ ) y se revisaron las atribuciones de la Secretaría de Energía, situación que detonó en una menor capacidad de gestión de la petrolera, pues quedó sujeta a mayor supervisión, control y seguimiento gubernamental, ${ }^{13}$ lo que a su vez incidió sobre las ya complicaciones en la toma de decisiones de los proyectos de la petrolera, y en particular en las de Cantarell.

Tan sólo hacia 2004, el complejo Cantarell era operado por 221 empresas, de las cuales más del 90\% eran de origen extranjero (Pérez, 2004).

Entre las medidas adoptadas destacaron: probar nuevos horizontes de producción; el desarrollo de campos adyacentes como Sihil, Kutz, Ixtoc y Kambesah para aprovechar la infraestructura existente; establecer estrategias diferenciadas de explotación con base en las condiciones geológicas del yacimiento; incorporar nueva infraestructura para el manejo de la sal en las actividades de explotación; la construcción e instalación de plataformas de perforación adicionales; el uso de nuevas tecnologías en la perforación (pozos no convencionales) y la terminación de pozos, para producir en zonas de espesor reducido (SENER, 2011: 103).

Por ejemplo, Pemex programó niveles de producción de petróleo en el complejo Cantarell de 1.9, 1.68 y 1.43 mmbd, para los ańos 2006, 2007 y 2008, lo que contrastó con los valores observados de 1.8, 1.4 y $1 \mathrm{mmbd}$, respectivamente (Pemex, 2005).

Hasta antes de la reforma energética, todas las operaciones de adquisición de obras, materiales y servicios debía apegarse al esquema legal único definido para todo el gobierno, lo que retrasaba las operaciones de compra de Pemex.

Cantarell fue una de las primeras áreas de trabajo en donde la CNH puso su atención a fin de atender la problemática enfrentada. 


\section{CANTARELL EN LA ACTUALIDAD}

A mediados de 2014, el Activo Cantarell ${ }^{14}$ estaba constituido por los campos Nohoch, Chac, Akal, Kutz, Ixtoc, Takín, Ek, Balam, Sihil, Kambesah y Után, de los cuales el último no estaba produciendo. A excepción de los campos Kambesah e Ixtoc, produce petróleo pesado que va de los 12 y $24^{\circ}$ API (American Petroleum Institute), con alto contenido de azufre y metales. La roca almacén es una brecha de clastos de caliza y carbonatos dolomitizados, naturalmente fracturada con alta permeabilidad y porosidad.

En general, los campos de Cantarell cuentan con alta permeabilidad de las rocas, amplios espesores de roca impregnada y están ubicados en tirantes de agua no mayores a 100 metros y a profundidades por arriba de hasta más de 3 mil metros. Se considera como un yacimiento maduro, por lo que su producción de aceite ha venido obteniéndose con un alto porcentaje de agua y un acelerado avance del contacto de gas, lo que ha propiciado una menor ventana de aceite (Morales et al., 2014), las cuales varían de 60 a 100 metros.

El volumen original de hidrocarburos contenido en el Activo Cantarell a finales de 2013 se ubicó en 42.6 miles de millones de barriles de petróleo crudo equivalente (mmmbpe), un nivel $2.1 \%$ inferior al registrado en 2002. Lo anterior, permite clasificarlo como un campo supergigante (Ivanhoe y Leckie, 1993: 87-91), no obstante la $\mathrm{CNH}$ ha determinado que se trata de un campo "compartamelizado" (Comisión Nacional de Hidrocarburos, 2013: 87).

De igual manera, al cierre de 2013, el total de reservas 3P de hidrocarburos, que se ubicó en $5.3 \mathrm{mmmbpce}$, registró una reducción del $53 \%$ respecto al reportado en el año 2002. No obstante, lo más alarmante fue el comportamiento de las reservas $1 \mathrm{P}$ que disminuyeron de 9.0 a $2.2 \mathrm{mmmbpce}$, situación que va en sentido contrario a la tendencia internacional de que el crecimiento de las reservas tiende a ser mayor en los campos más grandes, más viejos, particularmente de los ubicados costa adentro (UK Energy Research Centre, 2009: 58). El deterioro en las reservas probadas en Cantarell ha sido reflejo de la producción obtenida, pero también de los problemas en la administra-

La denominación de Cantarell se definió en inicio como los campos Akal y Nohoch. Años siguientes se definió el Complejo Cantarell que también comprendió los campos Kutz, Chac y Sihil, luego se incorporaron los campos Ixtoc y Takín cuando se definió el Proyecto Cantarell. Después, se adicionaron los campos Ek y Balam en el denominado Activo Integral Cantarell. Finalmente, a este último se incorporaron los campos Kambesah y Után. 
ción del yacimiento y su proceso de agotamiento, ${ }^{15}$ que se ha traducido entre otros factores por la invasión de agua. A su vez, la caída en las reservas fue fundamental para impulsar la diversificación de la cartera de proyectos de la petrolera estatal, entre las que destacan su incursión en las operaciones en aguas profundas, campos maduros y complejos como el caso del proyecto del Aceite Terciario del Golfo (antes Chicontepec).

Del total de reservas probadas de aceite, líquidos y condensados, las localizadas en el campo Akal fueron las más importantes al cierre de 2013 con el $75 \%$ del total del Activo Cantarell y una contribución del $16.9 \%$ de total nacional, pero además con un potencial de reservas $2 \mathrm{P}$ y $3 \mathrm{P}$, sin embargo, es el que más pérdida de reservas probadas ha experimentado a lo largo de los años.

El segundo campo más importante en reservas de petróleo fue Sihil, seguido por Ek, Ixtoc y Kutz que acumularon en conjunto casi una quinta parte de todas las reservas $1 \mathrm{P}$ del Complejo. Por su parte, los de mayor reservas totales (3P) fueron el primero y segundo de ellos más el campo Balam. No existe posibilidad de cambiar la tendencia declinante de la reservas remanente de hidrocarburos en Cantarell, a menos que se descubriera un bloque autónomo. Entre 2001 y 2012 los descubrimientos se reportaron en tres pozos Után-1, Sihil-5 y el Ixtoc-22 y sumaron $223 \mathrm{mmbp}$ de reservas 3P, de los cuales un poco más de la mitad correspondieron a reservas $1 \mathrm{P}-102.2 \mathrm{mmb}$ de aceite y $73 \mathrm{mmmpc}$ a gas natural-. Tampoco se ha registrado la incorporación de grandes volúmenes reservas probadas por efecto de revisiones.

Hacia finales septiembre de 2014 , la producción de petróleo crudo en el Activo Cantarell fue de $351 \mathrm{mbpd}$, siendo el campo Akal el que agudizó su agotamiento más rápido de lo planeado (Luhnow, 2007). Por lo anterior, su contribución en la producción nacional de petróleo se desplomó de un máximo en marzo de 2005 de 65.5 a 14.7\% en ese septiembre de 2014. Las adicionales producciones de petróleo en los campos Sihil, ${ }^{16}$ Ek e Ixtoc, resultado del aumento en el número de pozos productores -especialmente en los dos primeros casos desde 2009-, ${ }^{17}$ compensaron relativamente la caída del

15

16

17

El número de pozos productores aumentó entre enero de 2009 y diciembre de 2012 de 7 a 15 en el caso del campo Ek y de 5 a 20 en el caso del Sihil. Comisión Nacional de Hidrocarburos, www. cnh.gob.mx, 12/3/2012 
campo Akal. Estos tres campos llegaron a contribuir con alrededor del 43\% del total de la producción del crudo del complejo, luego de que en 2004 sólo aportaron menos del 1\%. En tanto, los campos Chac, Balam y Kutz observaron un proceso de agotamiento (véase cuadro 1 ).

En suma, a mediados de 2014 el Activo Cantarell aportó alrededor del $13 \%$ del total de crudo producido en México y una tercera parte del total de crudo pesado, y participó con el $34.2 \%$ de toda la producción acumulada histórica de aceite en el país al cierre de 2013.

Cuadro 1. Producción de petróleo en los Campos de Cantarell.. Miles de barriles por día

\begin{tabular}{|c|c|c|c|c|c|c|c|c|c|c|}
\hline Año & Cantarell & Akal & Balam & Chac & $E k$ & Ixtoc & Kutz & Nohoch & Sihil & Takín \\
\hline Origen a 1989 & 843 & 852 & & & & 5.8 & & 58 & & \\
\hline 1990-1999 & 843 & 994 & 24 & 38 & 9 & 8 & & 73 & & \\
\hline 2000 & 1471 & 1361 & 18 & 18 & 3 & 12 & & 59 & & \\
\hline 2001 & 1731 & 1604 & 20 & 22 & 2 & 11 & 14 & 68 & & \\
\hline 2002 & 1901 & 1805 & 13 & 17 & 1 & 11 & 9 & 46 & 2 & \\
\hline 2003 & 2122 & 2028 & 14 & 20 & 1 & 11 & 13 & 26 & 9 & \\
\hline 2004 & 2137 & 2038 & 11 & 17 & 1 & 11 & 12 & 41 & 6 & \\
\hline 2005 & 2036 & 1938 & 6 & 12 & 2 & 13 & 12 & 36 & 19 & \\
\hline 2006 & 1802 & 1701 & 7 & 12 & 6 & 14 & 12 & 34 & 16 & \\
\hline 2007 & 1491 & 1389 & 10 & 13 & 17 & 12 & 11 & 28 & 12 & \\
\hline 2008 & 1040 & 925 & 12 & 12 & 18 & 12 & 11 & 24 & 19 & 7 \\
\hline 2009 & 685 & 543 & 10 & 12 & 28 & 11 & 10 & 18 & 35 & 18 \\
\hline 2010 & 558 & 370 & 7 & 14 & 50 & 11 & 21 & 14 & 60 & 11 \\
\hline 2011 & 501 & 314 & 5 & 9 & 47 & 15 & 19 & 12 & 66 & 14 \\
\hline 2012 & 454 & 234 & 7 & 6 & 42 & 23 & 13 & 6 & 110 & 12 \\
\hline 2013 & 440 & 203 & 9 & 10 & 51 & 29 & 3 & 8 & 110 & 8 \\
\hline
\end{tabular}

* Se consideran todos los campos que reportan en el que Pemex denomina Activo Cantarell para fines administrativos. Nota. Hasta el año 2013, Kambesah produjo 10 mil barriles por día y Után no producía, pues sólo contaba con reservas de gas natural.

Fuente: CNH. 
El patrón de explotación de petróleo en Cantarell no ha seguido el de otros campos gigantes de tener un periodo prolongado de meseta (ASPO, 2001: 5), ya que su tasa anual de declinación fue de $20.3 \%$ entre 2006 y 2011 , lo cual contrastó con la práctica internacional en ese tipo de macro yacimientos (de entre 4 y 6\%) o la de los ubicados costa fuera que se estimó en 10\% (Höök, 2009: 2268). ${ }^{18}$ Pemex justificó la baja en la producción en Cantarell asociada a su etapa de madurez, en virtud "... del incremento en el porcentaje de agua y contenido de sal en las corrientes de crudo... (a que)... los espesores de aceite en el campo son cada vez más reducidos y en algunas partes, se elevan canalizaciones de gas y de agua en los pozos...(y al)... efecto de la extracción de gas de zona de transición en la producción de agua y aceite..." (Pemex PeP, 2012a: 5) situación que llevó al cierre de pozos ${ }^{19}$ y a crear infraestructura para el manejo de esa agua.

El factor de recuperación de aceite de Cantarell, definido como la relación producción acumulada a volumen original de reservas totales (3P), fue de $36.4 \%$ al cierre de 2013 nivel similar al del promedio mundial de 35\% (Babadagli, 2007: 222) y uno de los más elevados en el país, ${ }^{20}$ pero se aceleró desde 2002, año en que se ubicó en $23.5 \%$ por efecto del aumento de la producción y la menor incorporación de reservas.

La producción de gas natural en el Activo Cantarell mantuvo una relación por barril de petróleo extraído estable desde los inicios de su explotación hasta el año 2006, equivalente a $7.4 \%$ de gas natural por cada barril de petróleo obtenido -medidos en barriles de petróleo crudo equivalente-, pero desde 2007 comenzó a elevarse a 34\% hasta alcanzar un promedio de 39\% entre 2009 y 2013, situación atribuible a la citada decisión de inyección del gas nitrógeno y la propia reinyección de gas amargo que se inició desde julio de 2004. Así, la producción de gas natural asociado se fue elevando paulatinamente, de 566 mmpcd en el año 2000 a un máximo de 1627 mmpcd en 2008, para culminar durante 2013 en un 1002 mmpcd.

18

19

20

Existen otros cálculos por ejemplo Cambridge Energy Research Associates estimó 4.5\%, ExxonMobil entre 4 y 6\% y la International Energy Agency 6.7\% (Höök et al., 2009: 2262).

Entre 2011 y 2012 se llegó a producir un volumen promedio de hasta 101 mbd, después de promediar 62 mbd entre 2008 y 2010 (Comisión Nacional de Hidrocarburos, 2013).

Hacia 2008, Akal contaba con el segundo más importante factor de recuperación de aceite del país con el 35.3\%, por debajo del campo Abkatún-Pol-Chuc que registró el mayor nivel en el país con $37.5 \%$. Le siguieron Nohoch con $29.7 \%$, y por muy detrás del resto de campos ubicados en Cantarell (Comisión Nacional de Hidrocarburos, 2010). 
No obstante, mucha de la producción fue de gas nitrógeno, pues la relación de éste a gas natural producido pasó de $18 \%$ en 2007 a 148\% en 2013. Pemex no estuvo preparado para soportar el aumento en la producción de gas, porque no contaba con la infraestructura adecuada para su total aprovechamiento, ni para separarlo del nitrógeno, situación que originó dos problemas:

1) La contaminación del gas natural -con el nitrógeno- generó presiones de los grandes consumidores para que se les concedieran descuentos a los precios de primera mano. Ante tal petición, y con dos años de retraso en su entrada en operación, ${ }^{21}$ Pemex tuvo que desarrollar esquemas para el manejo de nitrógeno y construir una planta eliminadora del mismo en el Complejo Procesador de Gas Ciudad Pemex, Tabasco con capacidad de procesamiento de $630 \mathrm{mmpcd}^{22}$

2) Fue la creciente quema de gas a la atmósfera desde el $2001,{ }^{23}$ que se fue agudizando con el tiempo, ${ }^{24}$ por lo que la Comisión Nacional de Hidrocarburos ( $\mathrm{CNH}$ ) manifestó que "se puso en riesgo" la óptima explotación de Cantarell. ${ }^{25}$ Este problema implicó para Pemex no sólo un costo de oportunidad de aprovechar el gas natural, sino que incurrió en gastos porque al gas desperdiciado se le aplicó el mismo impuesto que al canalizado a la venta, situación poco razonable puesto que el gobierno fue el que no decidió invertir en infraestructura para su manejo.

En 2004 se anunció que la planta entraría en operación en diciembre de 2006 e inició hasta finales de 2008 .

Esta planta tuvo un costo de un poco más de 150 millones de dólares y su objetivo fue el de tratar el gas con concentraciones de nitrógeno de hasta $19.1 \%$ disminuyéndolas a $1.2 \%$, para cumplir con la Norma Oficial Mexicana 001-SECRE-2003.

Desde el año 2001, Pemex registró un índice de aprovechamiento de $92.3 \%$ contra $98 \%$ que contemplan los estándares internacionales.

De acuerdo a un documento elaborado por el presidente del Grupo Constitución de 1917, el volumen de quema de gas ha sido elevado, alcanzando la cifras de 2001: 85 mil; en 2002: 63 600; en 2003: 52 mil; en 2004: 36 mil; en 2005: 38 400; en 2006: 57 200; en 2007: 112 mil; en 2008: 266 mil; en 2009: 206 mil; y en 2010: 120 mil barriles de petróleo crudo equivalente (Garaicochea, 2013: 1-5).

Según la Comisión Nacional de Hidrocarburos (CNH), Pemex puso en riesgo la óptima explotación de petróleo crudo en Cantarell derivado de la sobreexplotación que realizó entre 2010 y 2011, sobre todo por la cantidad de gas enviado a la atmósfera y quemado. La dependencia estimó que la paraestatal dejó de extraer cerca de 106 mil barriles al día de petróleo, debido a que contaba con la forma de aprovechar el gas que expide el petróleo (Comisión Nacional de Hidrocarburos, 2011). 
A partir de 2007, se fueron presentado mayores niveles de quema y venteo de gas en un contexto de precios altos del combustible, alcanzándose un nivel máximo de aprovechamiento de $65.8 \%$ en 2009 , esto fue equivalente a un total de gas no aprovechado de alrededor de 680 millones de pies cúbicos diarios (mmpcd). Ante tal problemática, la $\mathrm{CNH}$ aprobó y publicó disposiciones técnicas para evitar o reducir la quema y el venteo de gas en los trabajos de exploración y explotación de hidrocarburos. ${ }^{26}$ Derivado de ello, la petrolera estatal tuvo que construir infraestructura adicional para la recuperación del gas o su reinyección al yacimiento, entre otras medidas importantes. A partir de octubre de 2011, se logró cumplir con la meta del índice de aprovechamiento de gas de $96.6 \%$ que fijó la $\mathrm{CNH}$, lográndose un valor de $97.5 \%$, al cierre de diciembre de 2012, empero en un contexto de una menor producción del gas natural. Se estima que las pérdidas por la quema de gas en el Complejo Cantarell fueron de alrededor de 1.3 miles de mmd entre 2009 y 2012. ${ }^{27}$

El valor total de los hidrocarburos obtenidos en el Activo Cantarell hasta el cierre de 2013 fue de un aproximado de 445 miles de mmd, lo que significó importantes recursos de aportación a la renta petrolera nacional, ${ }^{28}$ ya que los costos de producción fueron bajos desde los inicios de su explotación hasta principios de la década pasada. En efecto, el costo original de producción fue de 2.50 dólares por barril (db), y a partir de 2000, con los procesos de recuperación instrumentados, se elevó de entre 3.50 y $4.50 \mathrm{db}$, hasta promediar un poco más de $7.7 \mathrm{db}$ entre 2010 y 2012. ${ }^{29}$ También encareció las operaciones, la complejidad para acceder a adicionales hidrocarburos y la infraestructura requerida para ello. ${ }^{30}$

26

La norma para disminuir la quema de gas a la atmósfera -particularmente en Cantarell- se publicó en el Diario Oficial de la Federación por la Comisión Nacional de Hidrocarburos el 4 de diciembre de 2009. Entre 2010 y 2011, las metas de aprovechamiento del gas no se cumplieron y para 2012, se estableció como meta alcanzar el $97.5 \%$ de aprovechamiento de gas promedio conforme a la Resolución CNH.E.07.001/11 de la CNH.

Estimado con información pública de Pemex Exploración y Producción. Región Marina Noreste, Documento Análisis Costo Beneficio, Proyecto Cantarell, Cambio de Monto y Alcance, México, octubre de 2012.

Se llegó a señalar una tasa interna de retorno del proyecto superior al 900\%, de acuerdo a información de la Secretaría de Energía (Comisión Económica para América Latina, 2001: 16).

Corresponde al costo de producción promedio en el campo Cantarell reportado por Pemex a la Security Exchange Commission.

Entre la que destacó un mayor número de pozos, reparaciones mayores y menores, adicionales plataformas, equipos para producir con el sistema de levantamiento artificial (bombeo neumático continuo) en muchos de los pozos y el tendido de kilómetros de ductos, entre otros, así como la erogación de gastos de operación e investigación. 
Del valor de los ingresos obtenidos a lo largo de la vida del Activo Cantarell, cerca del 73\% fueron se realizaron entre los años 1998 y 2013, periodo en el que se invirtieron alrededor de $42 \mathrm{mil} \mathrm{mmd,} \mathrm{lo} \mathrm{que} \mathrm{significó} \mathrm{una} \mathrm{proporción}$ de un poco más de 7.3 dólares de ingreso por cada uno de inversión.

Cabe destacar, que considerando el patrón de comportamiento de un campo costa fuera, entre 2004 y 2012, la paraestatal dejó de percibir algo así como $70 \mathrm{mmmd}$ (véase gráfica 3), ${ }^{31}$ en virtud de que su tasa de declinación fue superior a la de otros grandes campos.

Por otra parte, durante toda la vida de explotación de Cantarell no hubo una estrategia óptima de administración del yacimiento en función de las condiciones de oferta y demanda en el mercado petrolero internacional, pues se explotó sin optimizar los ingresos ante la oportunidad de precios atractivos, siendo la década de los noventa cuando se registró la peor correlación.

\section{¿HACIA DÓNDE VA CANTARELL?}

Aunque existe el agotamiento de muchos campos petroleros en el mundo, se estima que aún hay volúmenes considerables de hidrocarburos por recuperar, pues sólo el $1 \%$ extra en ese tipo de campos equivaldría a entre 20 y 30 mmmb adicionales. ${ }^{32}$

Hacia finales de 2013, el volumen original de reservas de hidrocarburos disponible en Cantarell se integró en gran parte de petróleo (alrededor del $92 \%)$. Su importancia radicó en que representó $14.7 \%$ del total nacional del volumen original de reservas de aceite, esto es la tercer área con mayores recursos después de los contenidos en Aceite Terciario del Golfo (30.7\%) y Ku-Maloop-Zaap (15\%), además de contar con más de una quinta parte de las reservas de aceite 1P y 16\% de las reservas 3P.

Si bien el potencial productor de Cantarell se ha explotado, especialmente en los campos Akal y Chac, todavía quedan reservas de hidrocarburos por extraerse, si se toman en cuenta los factores de recuperación de otras naciones,

Se calculó a partir de una tasa de declinación de 6\%, que es el límite superior del rango estimulado como práctica internacional y se comparó con el valor de la producción real de hidrocarburos. Todo ello a partir de 2004, en donde Cantarell alcanzó su pico de producción de petróleo. Además, se consideró la producción de gas real y su valuación a precios de mercado.

De hecho, estudios de la Agencia Internacional de Energía señalan que 20\% de la producción de petróleo del mundo en el año 2030 provendrá del uso de métodos de recuperación secundaria o terciaria, donde se clasifica a Cantarell (Comisión Nacional de Hidrocarburos, 2012: 10). 
Gráfica 3. Cantarell: Ingresos Obtenidos vs. Ingresos Potenciales* /

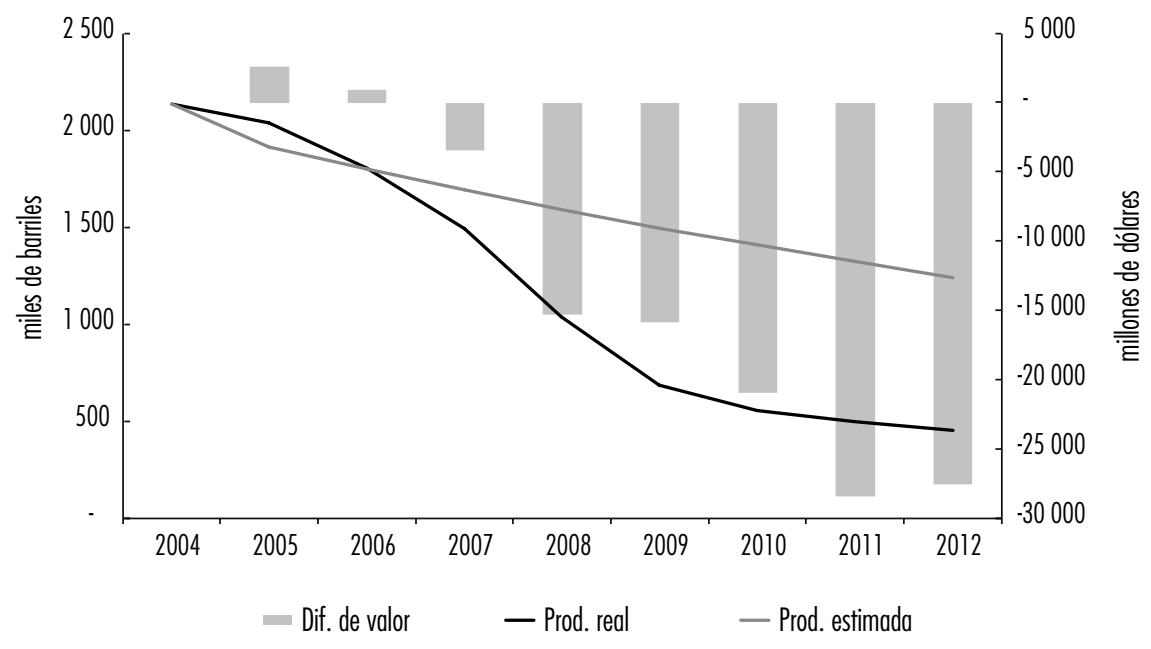

* / Se refiere a la comparación entre la valuación de los volúmenes obtenidos contra los que se determinarían a partir de las prácticas internacionales de una tasa de declinación de 6\%.

Fuente: elaborado con datos de Pemex.

en donde se logran obtener hasta el 46\% de recuperación (Jan Kjärstad, 2009: 451) o de los alcanzados en el Mar del Norte. De hecho, Pemex contempla recuperar entre 2013-2050 un volumen de aceite de 2,641 $\mathrm{mmb}$ de aceite y $1,436 \mathrm{mmmpc}$ de gas natural, para lo cual pretende invertir alrededor de 35 mmmd los proyectos Cantarell y Ek-Balam. ${ }^{33}$

Además de continuar explotando Akal, que cuenta con la mayoría de las reservas probadas de aceite, existe potencial en los campos Sihil, Ek, Balam y Kambesan, que contienen el $17 \%$ de la reserva probada del Complejo y una producción acumulada reducida. Los campos Kutz, Ixtoc y Nohoch contienen un $6 \%$ de esa reserva probada total de aceite y presentan también cierto potencial, ya que su factor de recuperación fue menor de $20 \%$ (véase cuadro 2).

Entre los retos planteados para continuar explotando hidrocarburos en Cantarell se han señalado los siguientes: incrementar el conocimiento sobre la caracterización de los campos, en particular la del daño a la formación en turas marinas y 31 ductos, un conjunto de reparaciones mayores y menores y el mantenimiento de la infraestructura (Pemex PEP, 2012a: 54). 
Cuadro 2. Reservas Probadas (1P) en Cantarell, 2013

\begin{tabular}{|c|c|c|c|c|c|c|}
\hline \multirow[t]{2}{*}{ Campo } & \multicolumn{2}{|c|}{ Reserva remanente IP } & \multicolumn{2}{|c|}{ Aceite, liq. y condensados } & \multicolumn{2}{|c|}{ Gas natural } \\
\hline & mmpce & $\begin{array}{c}\text { Factor } \\
\text { recuperación * }\end{array}$ & $\mathrm{mmbp}$ & $\begin{array}{c}\text { Factor } \\
\text { recuperación* }\end{array}$ & mmmpc & $\begin{array}{c}\text { Factor } \\
\text { recuperación }\end{array}$ \\
\hline Akal & 1666.2 & $42.2 \%$ & 1467.10 & $41.5 \%$ & 1037.9 & $50.2 \%$ \\
\hline Balam & 60.3 & $10.2 \%$ & 57.2 & $10.3 \%$ & 15.7 & $9.6 \%$ \\
\hline Chac & 20.3 & $34.1 \%$ & 18.8 & $34.3 \%$ & 7.8 & $31.6 \%$ \\
\hline Ek & 127.8 & $11.3 \%$ & 124.3 & $11.3 \%$ & 18.4 & $9.3 \%$ \\
\hline Ixtoc & 75.5 & $17.0 \%$ & 62.4 & $17.0 \%$ & 68.3 & $17.1 \%$ \\
\hline Kambesah & 29.4 & $2.4 \%$ & 26.3 & $2.5 \%$ & 16.2 & $1.7 \%$ \\
\hline Kutz & 8.6 & $16.2 \%$ & 7.9 & $16.3 \%$ & 3.6 & $15.2 \%$ \\
\hline Nohoch & 14.5 & $30.6 \%$ & 13.5 & $30.7 \%$ & 5 & $29.7 \%$ \\
\hline Sihil & 176.2 & $8.1 \%$ & 165.7 & $8.1 \%$ & 55.2 & $8.8 \%$ \\
\hline Takín & 7.8 & $22.2 \%$ & 7.6 & $22.0 \%$ & 1.3 & $35.3 \%$ \\
\hline Után & 2.8 & $0.0 \%$ & - & n. s. & 14.4 & $0.0 \%$ \\
\hline Total & 2189.4 & $37.1 \%$ & 1950.9 & $36.4 \%$ & 1244.0 & $44.0 \%$ \\
\hline
\end{tabular}

* Se refiere al factor de recuperación de las reservas 3 P entre su volumen original; n. a.: no aplica; n. s.: no significativo; mmbpce: millones de petróleo crudo equivalente; mmbp: millones de barriles de petróleo; mmmpc: miles de millones de pies cúbicos.

Fuente: elaborada con datos de la CNH.

yacimientos de crudo; mejorar la administración del contacto con el agua, particularmente en el campo Akal; integrar nuevas áreas de producción; neutralizar los efectos de la producción de gas en los pozos; optimizar el manejo y disposición de la propia agua y gas producido; la implementación de un proceso de recuperación secundaria mediante el doble desplazamiento para el campo Akal, así como elevar la eficiencia en la administración de las instalaciones que operaron volúmenes de producción hasta cinco veces superior a la obtenida a finales de 2012. Por ejemplo, en el campo Akal existen estimaciones (Rodríguez de la Garza, 2013), que señalan que podría elevarse la recuperación del aceite contenido en un 10\% adicional. Sin embargo, el punto crítico para aumentar el factor de recuperación es la rentabilidad de las operaciones, que a su vez, estará determinado por la eficiencia, productividad y por el nivel de los precios del petróleo en los mercados internacionales. 
De acuerdo a la metodología utilizada de emplear un modelo exponencial de declinación (Arps, 1944: 228-247), y con base en el uso de ecuaciones de hasta tercer grado, se calculó el pronóstico de producción de Cantarell hasta el año 2025 (véase cuadro 3). Los resultados comparativos de las estimaciones (véase gráfica 4) con respecto a los reportados por Pemex PEP son los siguientes: en ambos casos se muestra la tendencia declinante de la producción; sin embargo, se obtiene una menor velocidad en la caída de producción de petróleo esperada por Pemex, asociada al posible éxito en las estrategias de explotación aplicadas e inversiones realizadas en los últimos años, particularmente en el campo Akal, y potencial uso de técnicas de recuperación mejorada por aplicar.

Cuadro 3. Criterios Base para las Proyecciones de Producción de Aceite de los Campos de Cantarell

\begin{tabular}{|c|c|c|c|c|}
\hline \multirow[t]{2}{*}{ Campo } & \multicolumn{2}{|c|}{ \% volumen original extraído } & \multirow[t]{2}{*}{ Función o argumento de cálculo } & \multirow[t]{2}{*}{ Observación } \\
\hline & 2012 & 2025 & & \\
\hline Akal & 34 & 35 & $y=14.905 e-0.092 x$ & $R^{2}=0.8284$ \\
\hline Sihil & 7 & 24 & Tasa de declinación anual de $11 \%$ desde 2014 & \\
\hline Hohoch & 19 & 20 & $y=37.599 e-0.154 x$ & $R^{2}=0.8901$ \\
\hline Kutz & 17 & 22 & $y=10.328 \mathrm{e}-0.252 x$ & $R^{2}=0.8692$ \\
\hline Ixtoc & 18 & 26 & $y=0.0155 x+4.755$ & \\
\hline Ek & 10 & 27 & $y=-0.1445 x 2+3.7358 x-5.7058$ & \\
\hline Chac & 36 & 40 & $y=14.905 e-0.092 x$ & $R^{2}=0.8284$ \\
\hline Balam & 11 & 22 & $\begin{array}{l}y=-0.0003 \times 4+0.0173 \times 3-0.3148 \times 2+ \\
1.459 x+7.4792\end{array}$ & $\begin{array}{l}\text { Función ajustado a un } \\
\text { volumen de } 112 \mathrm{mmb}\end{array}$ \\
\hline Kambesah & $0 \%$ & n.d. & n.d. & n.d. \\
\hline
\end{tabular}

n.d.: no disponible.

Fuente: elaboración propia con datos de Pemex. 
Gráfica 4. Pronóstico de Producción de Petróleo en Cantarell. Miles de barriles por día

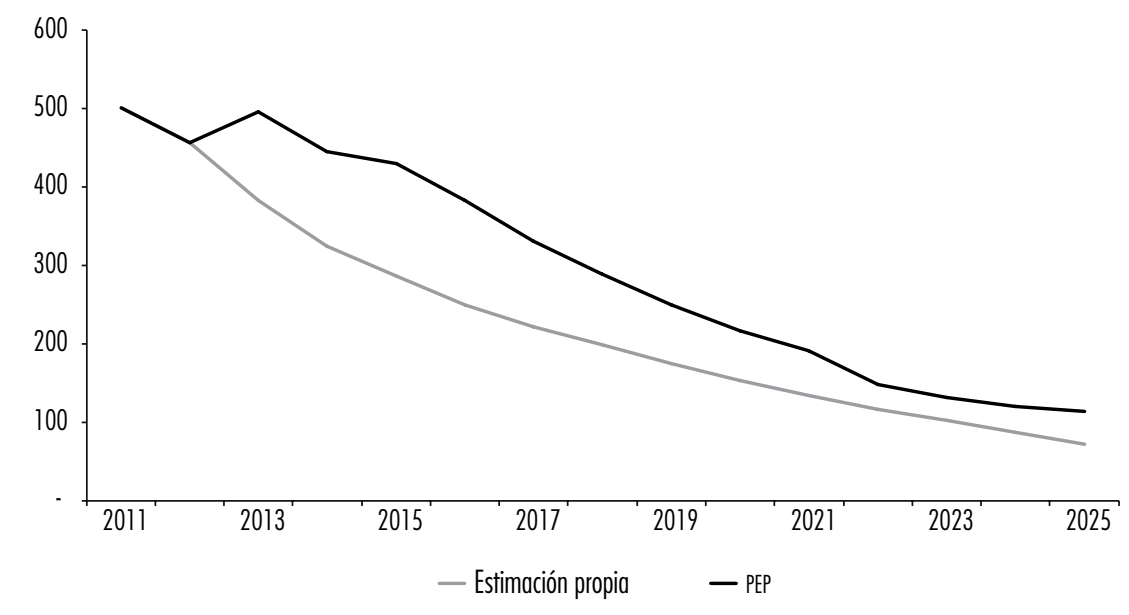

Fuente: Pemex PEP y estimaciones propias.

\section{CONCLUSIONES}

Cantarell, y particularmente el campo Akal, ha sido una de las fuentes de renta petrolera más importante para México, que ha incidido positivamente sobre la oferta de energía primaria, la captación de ingresos de divisas por los volúmenes de crudo Maya exportado, amén de los conocimientos y experiencia para la industria petrolera, y para Pemex en particular.

Cantarell llegó a tener un rol relevante como proveedor de petróleo pesado en el mundo hasta mediados de la década pasada y a ser considerado como un campo supergigante. Desde sus inicios ha sido un destino prioritario de las inversiones en la industria petrolera ante su elevada rentabilidad, debido a sus bajos costos de operación y la amplia disponibilidad de reservas de petróleo y gas natural. Sin embargo, sus operaciones han estado sujetas a decisiones gubernamentales y corporativas que han incidido sobre su óptima explotación. De manera particular, se pueden identificar tres etapas básicas en su desempeño, en donde el factor común ha sido el cubrir las necesidades financieras de los gobiernos en turno y el apoyo para mejorar la situación económica del país: 
1) Una primera etapa cubrió desde sus inicios de su explotación hasta finales de la década de los noventa. En ese lapso, las operaciones de Pemex se concentraron básicamente en la explotación de petróleo en Cantarell, con independencia de las condiciones de oferta y demanda del mercado petrolero internacional.

2) La otra etapa comprendió desde finales de los años noventa hasta el pico de petróleo en 2003. Se caracterizó porque el gobierno destinó adicionales recursos de inversión a Cantarell, y se instrumentaron distintas estrategias, entre las que destacó el empleo de nitrógeno para elevar su presión. La determinación del gobierno se modificó parcialmente ante el peligro de perder una fuente de aporte de ingresos.

3) Una última etapa comprende desde 2004, y en donde a pesar de los recursos de inversión canalizados se ha caracterizado por la declinación de los campos, particularmente de Akal, que restaron productividad, eficiencia, y sobre todo, mayores complicaciones para acceder al aceite.

La producción de petróleo en Cantarell continuará por lo menos en las siguientes dos décadas, extendiendo su proceso de declinación, el cual podría atenuarse con el uso de tecnología enfocada a ampliar el conocimiento geológico de los campos y su caracterización, robustecer los métodos de recuperación empleados -especialmente en el campo Akal-, amén de la canalización de adicionales recursos financieros.

En suma, la determinación de la explotar un campo petrolero del tamaño de Cantarell conviene realizarse dando prioridad a decisiones técnicas y estratégicas de mercado, y no a las relativas a las políticas gubernamentales de corto plazo. En particular, una de las incógnitas que quedan en la discusión es sobre la efectividad de la inyección de nitrógeno como mecanismo primordial para elevar la producción de petróleo. La rápida declinación en el volumen producido en Cantarell, representa una alerta en la administración de otros campos como KMz. 


\section{ANEXO 1}

Anexo 1. Relevancia del Campo Akal en México

\begin{tabular}{|c|c|c|c|c|c|c|c|}
\hline Concepto & $2000-2007$ & 2008 & 2009 & 2010 & 2011 & 2012 & 2013 \\
\hline Producción de aceite (mbd) & 1741 & 934 & 541 & 366 & 314 & 235 & 203 \\
\hline Producción de gas natural (mmpcd) & 688 & 1559 & 1397 & 1191 & 1014 & 926 & 921 \\
\hline Producción de agua (mbd) & 4 & 56 & 62 & 68 & 103 & 99 & 115 \\
\hline Pozos Operando (núm.) & 191 & 171 & 162 & 152 & 161 & 164 & 149 \\
\hline Producción aceite/pozo (mbd) & 9.1 & 5.5 & 3.3 & 2.4 & 2.0 & 1.4 & 1.4 \\
\hline Reservas IP Aceite, liq. y cond. (mmbd) & n.d & n.d & 2151 & 1839 & 1733 & 1629 & 1467 \\
\hline Reserva IP Gas nat. (mmpcd) & n.d & n.d & 1216 & 1469 & 1148 & 1092 & 1038 \\
\hline Reserva 1P/prod -aceite- (años) & n.d & n.d & 10.9 & 13.8 & 15.1 & 19.0 & 19.8 \\
\hline Relación gas/aceite (pc/bl) & 421 & 2977 & 5216 & 6364 & 5013 & 4889 & 6274 \\
\hline Prod. petróleo Akal a prod. total Cantarell & $94.8 \%$ & $89.8 \%$ & $78.9 \%$ & $65.6 \%$ & $62.7 \%$ & $51.7 \%$ & $53.4 \%$ \\
\hline $\begin{array}{l}\text { Prod. gas natural Akal a prod. total } \\
\text { en Cantarell }\end{array}$ & $93.2 \%$ & $96.8 \%$ & $99.7 \%$ & $97.3 \%$ & $94.4 \%$ & $92.3 \%$ & $91.9 \%$ \\
\hline $\begin{array}{l}\text { Reservas totales aceite 1P en Akal } \\
\text { a totales Cantarell }\end{array}$ & n.d & n.d & $72.7 \%$ & $72.4 \%$ & $74.0 \%$ & $69.1 \%$ & $75.2 \%$ \\
\hline $\begin{array}{l}\text { Reservas totales gas natural en Akal } \\
\text { IP a totales Cantarell }\end{array}$ & n.d & n.d & $61.1 \%$ & $89.3 \%$ & $88.2 \%$ & $82.3 \%$ & $83.4 \%$ \\
\hline
\end{tabular}

n. d.: no disponible; mbd: miles de barriles por día; mmpcd: millones de pies cúbicos diarios; pc/bl: relación Gas - Aceite es la cantidad de gas disuelta en el aceite en pies cúbicos por barril o pc/bl).

Fuente: elaboración propia con datos de Pemex y CNH.

\section{BIBLIOGRAFÍA}

Arps, J.J. (1944), "Analysis of Decline Curves", Transactions of the American Institute of Mining, Metallurgical and Petroleum Engineers, vol. 160, USA, pp. 228-247.

ASPO (2001), Analysis of the UK Oil Production, LB-Systemtechnik GmbH Daimlerstr. 15D-85521 Ottobrunn, documento, pp. 11, 22 ${ }^{\text {nd }}$ February (consultado el 12 de abril de 2014), disponible en <http://www.peakoil. net/.../06_Analysis_of_UK_oil_prod...>

Babadagli, Tayfun (2007), "Development of Mature Oil Fields -A Review", Journal of Petroleum Science and Engineering, 57, USA, pp. 221-246. 
Barton, R. et al. (2009/2010), "High-Impact Reservoirs", Oil Field Review, 21, núm. 4,USA, pp. 14-29.

Comisión Económica para América Latina y el Caribe (Cepal) (2001), Retos y posibles soluciones para el sector energético mexicano, LC/MEX/L.505, 20 de diciembre de 2001, p. 76.

Comisión Nacional de Hidrocarburos (2010), "Factores de recuperación de aceite y gas en México", Documento Técnico (DT-1) (consultado el 2 de septiembre de 2014), p. 116, disponible en <www.cnh.gob.mx>

(2011), Resolución CNH.04.001/11, Documento Técnico (consultado el 10 de octubre de 2014), disponible en <http://www.cnh.gob.mx>

(2012), "El futuro de la producción de aceite en México", Documento Técnico (consultado el 10 de julio de 2014), p. 126, disponible en <http:// www.cnh.gob.mx>

(2013), "Dictamen Técnico del Proyecto de Explotación Cantarell (Modificación Sustantiva)", Documento Técnico (consultado el 22 de octubre de 2014), p. 202, disponible en <www.cnh.gob.mx>

Garaicochea Petrirena, Francisco (2007), "La Explotación irracional de Cantarell”, Revista Petróleo y Electricidad, núm. 111, septiembre, México (consultado el 8 de mayo de 2014), disponible en <http://esp.mexico. $\mathrm{com} /$ revistas/index.php?idrevista $=70 \&$ idarticulo $=522>$

(2013), "Éxitos y fracasos de Pemex Exploración y Producción", Documento Técnico, 5 de abril (consultado el 15 de agosto de 2014), disponible en <http://grandesproblemas.org.mx/temas/ponencias/exitos-yfracasos-de-pemex-exploracion-y-produccion-pdf $>$

Höök, Mikael et al. (2009), "Giant Oil Field Decline Rates and their Influence on World Oil Production”, Energy Policy, vol. 37, USA, pp. 2262-2272.

Ivanhoe, L. y G. Leckie (1993), "Global Oil, Gas Fields, Sizes Tallied, Analyzed”, Oil and Gas Journal, February 15, USA, pp. 87-91.

Jan Kjärstad, Filip Johnsson (2009), "Resources and Future Supply of Oil", Energy Policy, vol.37, USA, pp. 441-464.

Lajous Vargas, Adrián (2009), "El ocaso de Cantarell", Revista Nexos, vol. 31, núm. 182, México, D.F.

Luhnow, D. (2007), “Mexico's Oil Output Cools”, Rigzone Magazine, January 29 (consultado el 4 de octubre de 2014), disponible en <http:// www.rigzone.com/news/article.asp?a_id=40538>

Morales, J, C. Pérez y H. Márquez (2014), "Modelado estático del campo Cantarell y verificación de predicciones con pozos recientemente perforados, Sonda de Campeche, México", Revista Ingeniería Petrolera, vol. 54, núm. 7, julio, México, pp. 376-384. 
Pemex (2005), "Precisiones respecto al comportamiento del complejo Cantarell”, Boletín de Prensa, Boletín No. 287, 8 de diciembre de 2005 (consultado el 12 de agosto de 2014), disponible en <http://www.pemex.com>

Pemex pep (2012a), Análisis costo beneficio Proyecto Integral Cantarell, documento versión publicable, pp. 69, octubre de 2012 (consultado el 15 de junio de 2014), disponible en <http://www.shcp.gob.mx>

Pemex PEP (2012b), "Incorporación de reservas de gas y aceite e incremento de producción”, Libro Blanco 2006-2012, p. 558.

Pérez, Ana L. (2004), "Cantarell daños Irreversibles", Revista Contralinea, artículo, marzo de 2004 (consultado el 19 julio de 2014), disponible en <http://contralinea.com.mx/archivo/2004/marzo/capitales/index.html>

Periódico El Economista (2012), "Pemex explota el pozo más profundo en la historia de Cantarell", nota periodística, 19 julio (consultado el 13 de enero de 2014), disponible en <http://eleconomista.com.mx/industrias/2012/07/19/pemex-explota-pozo-mas-profundo-historia-cantarell>

Robelius, F. (2007), Giant Oil Fields - The Highway to Oil, Giant Oil Fields and their Importance for Peak Oil. Licentiate thesis from Uppsala University, p. 168.

Rodríguez de la Garza, Fernando (2013), "”Retos de la recuperación mejorada y avanzada de crudo en la Sonda de Campeche", Instituto de Geofísica, presentación realizada el 23 de agosto de 2013 (consultado el día 12 de diciembre de 2015), disponible en <https://www.youtube.com/ watch? $=\mathrm{Nr} 6 y v \mathrm{G} 55 \mathrm{U}-\mathrm{o}>$

Romo Rico, Daniel (2010), "La política fiscal y su relación con el desempeño de Pemex, 1998-2008”, en Martínez María de Rosario et al., Libro Administración de Riesgos, vol. II, México, UAM, pp. 417-444.

(2011), Pemex, Origen, Evolución y Perspectivas, México, Pemex-IPN, p. 318.

Sener (2011), Prospectivo del Mercado de Petróleo Crudo, 2010-2025, México, p. 175.

(2003), Tercer Informe de Labores, México.

Sorrell Steve, Jamie Speirs et al. (2012), "Shaping the Global Oil Peak: A Review of the Evidence on Field Sizes, Reserve Growth, Decline Rates and Depletion Rates", Energy, vol. 37, USA, pp. 709-724.

UK Energy Research Centre (2009), An assessment of the evidence for a nearterm peak in global oil production, august (consultado el 4 de octubre de 2014), pp. 198, disponible en <http://aie.org.au/AIE/Documents/ Global_Oil_Depletion_UKERC.pdf> 